\title{
Equipos de Ayuda, Maltrato entre Iguales y Convivencia Escolar
}

\author{
Avilés Martínez, José María ${ }^{1}$ \\ Torres Vicente, Nieves ${ }^{2}$ \\ Vian Barón, Maria Victoria ${ }^{3}$
}

${ }^{1}$ Departamento de Psicología. Universidad de Valladolid ${ }^{2}$ Departamento de Orientación. IES Juana I de Castilla, Tordesillas (Valladolid) ${ }^{3}$ Departamento de Orientación. IES Julián Marías (Valladolid)

\section{España}

\section{aviles@uva.es}

Dirección postal: Facultad de Educación y Trabajo Social. Dpto. de Psicología. Campus Miguel Delibes s/n. 47011 Valladolid.

(C) Education \& Psychology I+D+i and Editorial EOS (Spain) 


\section{Resumen}

Introducción. Se exploran los efectos de un Programa de Ayuda entre Iguales (Cowie, 1998) como una medida, entre otras (amistad, mentoría, mediación o consejo), para mejorar la convivencia escolar y prevenir el maltrato entre iguales. Analiza sus posibles efectos sobre el bullying y la satisfacción de los diferentes sectores de la comunidad educativa sobre su puesta en práctica.

Método. El trabajo se lleva a cabo mediante la aplicación del programa en un centro de Educación Secundaria Obligatoria a través de Equipos de Ayuda. Se comparan sus efectos con los de una población control que no disfruta el programa, a partir de los datos del Insebull (Avilés y Elices, 2007), cuestionario sobre intimidación y maltrato entre iguales, que recoge la percepción que los escolares tienen de la incidencia y consecuencias del bullying sobre la convivencia escolar.

Resultados. No se obtienen diferencias en la incidencia del maltrato entre iguales en los centros comparados. Sin embargo, en el que aplica el Programa de Ayuda las víctimas elevan significativamente la comunicación de lo que les pasa; los agresores/as son más conscientes de sus actuaciones y el maltrato social se visualiza y considera significativamente más. Por sectores, el alumnado integrante de los Equipos de Ayuda considera que participar en el Programa ha modificado su forma de pensar y actuar; las familias de éstos, creen que es una experiencia muy positiva para sus hijos e hijas y defienden la permanencia de esta medida en el centro; el alumnado receptor del servicio valora positivamente su existencia; y el profesorado opina que los Equipos de Ayuda son capaces de realizar contribuciones significativas a la mejora de la convivencia del centro.

Discusión. Parece necesario combinar con los Programas de Ayuda otras iniciativas específicas de lucha contra el bullying en el marco de un Programa Antibullying en la comunidad educativa, de carácter colectivo, participado y duradero en el tiempo.

Palabras clave: Convivencia escolar, Bullying, Programas de ayuda, Equipos de ayuda, Comunidad educativa.

Recibido: 21/04/08 Aceptación inicial: 05/05/08 Aceptación final: 02/06/08 


\begin{abstract}
Introduction. We are exploring the effects of a Peer Support Programme (Cowie, 1998), as a means to, amongst others, (friendship, mentoring, meditation or advise), to improve school relationship and prevent the bullying. It analyzes its possible effects on bullying and the satisfaction of different sections of the teaching community when putting in practice.

Method. The work is carried out following a Programme in a Secondary school Centre through Support Teams. They compare their effects with those of a controlled population that did not undego the programme.The information gathered comes from CIMEI (Avilés 2005), a questionnaire on the intimidation and maltreatment amongst equals. The questionnaire gathers sdudents' opinions regarding the incidences and consequences of bullying on school living together.
\end{abstract}

Results. One does not find differences between incidences of bullying in the tested centres. However, when the Support Programme is undertaken, the victims significantly increase the communication with others regarding what is happening to them. Furthermore the agressors are more aware of the actions and the social maltreatment is significantly more considered and visualized. By sectors, the pupils taking part in the Support Teams consider that their participation in the programme has altered their way of thinking and acting. The families of the pupils tested, think that is a very positive experience for their sons and daughters and defend the measures in the Centre; the pupils value the existence of the service and the teachers believe that the Support Teams are capable of realizing significant contribution in the improvement of school living together.

Discussion. It seems necessary to combine peer support programmes with other specific antibullying innitiatives in the teaching community. They should be collective (nature), participative and long-term measures.

Key words: School living together, Bullying, Support Programmes, Support Teams, Teaching community,

Received: 21/04/08 Initial Acceptance: 05/05/08_Definitive Acceptance: 02/06/08 


\section{Introducción}

Los programas de ayuda se han revelado eficaces en general para trabajar la convivencia escolar de los grupos de iguales (Cowie y Fernández, 2006; Cowie y Jennifer, 2006; Cowie y Jennifer, 2007) y, en particular, para abordar cuestiones relacionadas con el problema del maltrato entre iguales -bullying- (Avilés, 2006a; Sharp y Smith, 1994; Smith y Sharp, 1994) en las relaciones interpersonales entre pares. Sin embargo, se hace necesario contrastar su posible influencia en la reducción de los índices de bullying en los grupos de iguales, los posibles cambios en la percepción de las distintas formas de maltrato y su contribución en la modificación de actuaciones de los perfiles, especialmente en víctimas y agresores. Además, nos interesa valorar su contribución en la percepción que de la mejora del clima de convivencia escolar, tienen los distintos sectores de la comunidad educativa.

Los programas de ayuda entre iguales en sus diferentes modalidades, ya sean de forma individual en la prestación de la ayuda (Fernández, Villoslada y Funes, 2002) o en Equipo de Ayuda -que es como presentamos este trabajo-, podemos enmarcarlos en los llamados Programas de Apoyo al alumnado (Avilés, 2006c) para la mejora de la convivencia escolar, donde caben múltiples fórmulas de ejecución. En la Tabla 1 puede verse esta diversidad.

En este marco, situamos los programas de ayuda entre los llamados "peer partnering" y "peer mentoring" (Sullivan, 2001). Por una parte, como una estrategia para llegar y estar con el alumnado nuevo o que puede estar en riesgo de sufrir maltrato, soledad, desorientación o dificultades de integración; y por otra, como un medio para proporcionar orientación, tutoría (incluso académica) y ayuda al alumnado que necesita una guía para afrontar satisfactoriamente las relaciones interpersonales (Trianes, 2005), o bien, ocupa roles grupales de riesgo (Salmivalli, Lagerspetz, Björkqvist, Österman, Kaukiainen, 1996). 
Tabla 1. Modalidades de Programas de Apoyo para la mejora de la Convivencia Escolar y la prevención del Maltrato entre Iguales.

\begin{tabular}{|c|c|c|c|c|}
\hline $\begin{array}{l}\frac{0}{0} \\
\frac{0}{0} \\
\sum\end{array}$ & $\begin{array}{c}\text { Amistad entre iguales } \\
\text { (Peer Partnering) }\end{array}$ & $\begin{array}{l}\text { Alumnado Mentor } \\
\text { (Peer Mentoring) }\end{array}$ & $\begin{array}{l}\text { Alumnado Mediador } \\
\text { (Peer Mediation) }\end{array}$ & $\begin{array}{l}\text { Alumnado Consejero } \\
\text { (Peer Counselling) }\end{array}$ \\
\hline 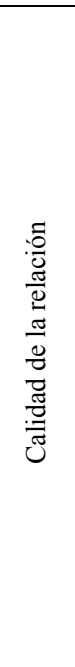 & $\begin{array}{c}\text { Relación de Colega } \\
\text { Compañía. } \\
\text { Amistad. } \\
\text { Compartir tiempos jugando o } \\
\text { hablando, ayudar a encontrar } \\
\text { soluciones, evitar la soledad y el } \\
\text { aislamiento. } \\
\text { Compartir sentimientos y pre- } \\
\text { ocupaciones. No culpabilizar. } \\
\text { Ofrecer apoyo, protección y } \\
\text { amistad. }\end{array}$ & \begin{tabular}{|} 
Relación de Mentor-Tutor \\
Tutorización. \\
Trabajo compartido. \\
Orientación. Guía. Modelo. \\
Ayuda en deberes. Debatir, \\
hablar, discutir, \\
reglas de funcionamiento \\
del grupo o escuela. \\
Fomentar confianza. Compartir \\
información.
\end{tabular} & $\begin{array}{l}\text { Relación de Mediador } \\
\text { Resolución de conflictos. } \\
\text { Mediación entre partes. } \\
\text { Ayudar en la búsqueda estruc- } \\
\text { turada y formal de: } \\
\text { Soluciones de las partes com- } \\
\text { partidas. } \\
\text { Relaciones más constructiva. } \\
\text { Alternativas satisfactorias. } \\
\text { Reducir los prejuicios. }\end{array}$ & $\begin{array}{l}\text { Relación Cliente-Consejero } \\
\text { Afrontamiento de problemas. } \\
\text { Información adecuada. } \\
\text { Contacto individual. } \\
\text { Tratamiento formal y supervi- } \\
\text { sado. Entender sentimientos. } \\
\text { Reflexionar en emociones. } \\
\text { Apoyar en decisiones. Orienta- } \\
\text { ciones. Feedback positivo. } \\
\text { Búsqueda de soluciones pro- } \\
\text { pias. Afrontar salidas al bull- } \\
\text { ying. }\end{array}$ \\
\hline 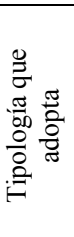 & $\begin{array}{c}\text { Alumnado acompañante } \\
\text { Hora del círculo- Circle Time } \\
\text { Amigos - Befrienders } \\
\text { Apadrinamientos - Buddies } \\
\text { Equipos de Ayuda }\end{array}$ & $\begin{array}{c}\text { Alumnado Tutor } \\
\text { Voluntariado inducido } \\
\text { Equipos de Ayuda } \\
\text { Teleayuda }\end{array}$ & $\begin{array}{l}\text { Mediadores escolares } \\
\text { Mediadores en clase } \\
\text { Alumnado Negociador } \\
\text { Ayudantes de recreo }\end{array}$ & $\begin{array}{c}\text { Alumnado Consejero } \\
\text { Teleayuda }\end{array}$ \\
\hline 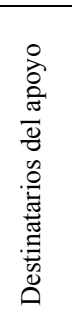 & $\begin{array}{c}\text { Alumnado con dificultades de } \\
\text { socialización o riesgo de agresión } \\
\text { Alumnado nuevo en el centro. } \\
\text { Alumnado con necesidad de } \\
\text { acompañamiento. } \\
\text { Casos de bullying incipiente. } \\
\text { Escuelas Primarias y Secundarias }\end{array}$ & \begin{tabular}{|} 
Alumnado con necesidad de \\
orientación y tutoría académica \\
y/o personal. \\
Alumnado con necesidad de \\
acompañamiento. \\
Escuelas primarias y secunda- \\
rias.
\end{tabular} & $\begin{array}{c}\text { Resolución de conflictos } \\
\text { Inducida por otros o buscada } \\
\text { por quienes están implicados. } \\
\text { Tercer ciclo de primaria y } \\
\text { escuelas secundarias. }\end{array}$ & $\begin{array}{l}\text { Resolución de conflictos. } \\
\text { Problemas de violencia. } \\
\text { Afrontamiento del bullying } \\
\text { desde cualquier rol. } \\
\text { Escuelas Secundarias. }\end{array}$ \\
\hline
\end{tabular}

En el caso que nos ocupa actualmente, hemos querido señalar de forma específica, la modalidad que designamos como Equipo de Ayuda, que supone un avance respecto a la concepción del servicio por parte de los adultos y en relación a la singularidad-colectividad de los alumnos, en comparación con el modelo más frecuente de alumnado ayudante. Aunque ambos participan de los mismos contenidos y actuaciones, en este último se hace más ostensible cómo la responsabilidad recae sobre los individuos que cumplen esa función y menos en un grupo. El concepto de Equipo de Ayuda parte de la idea de los equipos docentes del centro, de dotar a los grupos de iguales que conviven habitualmente juntos, de redes de apoyo estables, que actúan de forma cooperativa y colaborativa. El objetivo es primar la idea de equipo, de grupo de chicos/as que trabajan juntos, unidos por una misma actividad y que pueden ayudarse mutuamente en un momento determinado, independientemente de su edad. Se persigue que 
el alumno/a no se sienta solo/a ante la tarea de ayuda y, sobre todo, que aprenda a cooperar con otros en la ayuda. Frente a otras modalidades, la de Equipo de Ayuda aporta la idea, de grupo que busca un mismo fin y un sentimiento colectivo de apoyo, reforzando y evitando así la sensación excesiva de responsabilidad individual o, incluso, sobreprotagonismo (Avilés y Alonso, 2008; Sharp, Cowie y Smith, 1994). Por otra parte, contribuye a integrar cognitivamente estas habilidades, en el marco de las atribuciones que hacen los participantes y protagonistas en el fenómeno (Avilés, 2006b; Avilés y Mascarenhas, 2007). Incluso, puede llegar a suponer un ejemplo de actuación colectiva frente a determinadas situaciones de indefensión grupal provocadas por quienes pretenden ejercer el abuso.

\section{Objetivos de la investigación}

La investigación pretende diversos objetivos. Por una parte, establecer posibles diferencias en la incidencia del maltrato en los grupos que han recibido y puesto en práctica el programa de ayuda y los que no, y por otra, resaltar los posibles efectos de la aplicación del programa de ayuda entre los grupos de alumnos/as que lo llevan a cabo.

Así mismo, desde un punto de vista práctico, nos ha interesado diseñar una evaluación que abarque dos aspectos diferenciados: la valoración de la eficacia percibida del programa y la opinión de la comunidad educativa acerca de la contribución del programa en la mejora de la convivencia y del clima escolar, como han hecho otras investigaciones (Cowie y Fernández, 2006; Menesini, Codedcasa, Benelli y Cowie, 2003; Newman y Murray, 2005).

La valoración de la eficacia la consideramos directamente relacionada con el grado de satisfacción de los más directamente implicados en los Equipos de Ayuda, aspecto que queríamos conocer, para guiar la toma de decisiones posteriores sobre la intervención, especialmente en lo que a la continuidad y desarrollo del Programa se refiere. Este objetivo de evaluación, lo concretamos en diferentes dimensiones, a veces comunes a todos los evaluados, pero en otras, específicas para cada uno de ellos: información acerca del programa, adecuación de la formación impartida/recibida, consecuencias o efectos de la experiencia, consideración de la figura del Equipo de Ayuda, perspectivas sobre el programa, etc... 


\section{Método}

\section{Participantes}

La muestra estudiada ha sido de 136 chicos y chicas de entre 12 y 14 años del primer curso de la ESO de dos centros educativos de la provincia de Valladolid (Castilla y LeónEspaña). Uno participaba en un Programa de Ayuda y el otro no. Para la exploración se seleccionaron grupos completos de entre los que componían el primer nivel de la Etapa Secundaria Obligatoria de cada uno de los centros. La elección del nivel educativo vino motivada por ser primero de la ESO uno de los niveles donde la incidencia del bullying es más relevante y por tratarse de un alumnado que se incorpora por primera vez al centro educativo (aspecto coincidente en el grupo experimental y en el grupo control) y al programa de Equipos de Ayuda que luego continúa en cursos sucesivos (es el primer contacto que el grupo experimental tiene con los Equipos de Ayuda). Por tanto, toda la formación-información acerca del Programa que recibe el grupo experimental, tenemos la posibilidad de controlarla y establecerla desde el equipo de investigación. La distribución de la muestra por sexo y edad se recoge en las Tablas $\mathrm{n}^{\mathrm{o}} 2$ y 3 .

Tabla 2. Distribución por centro y sexo ${ }^{1}$

\begin{tabular}{|c|c|c|c|}
\hline Muestra & Chicos & Chicas & Total \\
\hline Centro 1 & $\mathbf{4 0}(58,8 \%)$ & $\mathbf{2 8}(41,2 \%)$ & $\mathbf{6 8}(100 \%)$ \\
\hline Centro 238 (58,5\%) & $\mathbf{2 7}(41,5 \%)$ & $\mathbf{6 5}(100 \%)$ \\
\hline Total & $\mathbf{7 8}(58,6 \%)$ & $\mathbf{5 5}(51,4 \%)$ & $\mathbf{1 3 3}(100 \%)$ \\
\hline
\end{tabular}

${ }^{1}$ Tres casos perdidos del sistema, por lo que la muestra total es de 133 chicos y chicas.

Tabla 3. Distribución por centro y edad ${ }^{2}$

\begin{tabular}{|c|c|c|c|c|}
\hline \multicolumn{4}{|c|}{ Edad } & Total \\
\hline \multirow{2}{*}{ Centro 1 } & 12 & 13 & 14 & \\
\cline { 2 - 5 } & $\mathbf{2 3}$ & $\mathbf{2 8}$ & $\mathbf{1 7}$ & $\mathbf{6 8}$ \\
\hline \multirow{2}{*}{ Centro 2 } & $33,8 \%$ & $41,2 \%$ & $25,0 \%$ & $100,0 \%$ \\
\cline { 2 - 5 } & $47,7 \%$ & $43,1 \%$ & $9,2 \%$ & $100,0 \%$ \\
\hline \multirow{2}{*}{ Total } & $\mathbf{5 4}$ & $\mathbf{5 6}$ & $\mathbf{2 3}$ & $\mathbf{1 3 3}$ \\
\cline { 2 - 5 } & $40,6 \%$ & $42,1 \%$ & $17,3 \%$ & $100,0 \%$ \\
\hline
\end{tabular}

${ }^{2}$ Tres casos perdidos del sistema, por lo que la muestra total es de 133 chicos y chicas. 
En el centro que realizó el Programa de Ayuda (centro 1) participaron en la evaluación: el alumnado integrante de los Equipos de Ayuda, el alumnado de $1^{\circ}, 2^{\circ}$ y $3^{\circ}$ de la ESO receptor del servicio de ayuda, los padres y madres del alumnado de los Equipos, profesorado de estos cursos y profesorado directamente implicado en la puesta en marcha y seguimiento del programa a lo largo del curso académico.

\section{Instrumento}

Se utilizaron diferentes instrumentos, uno para medir la incidencia y las dimensiones del maltrato entre iguales y otros para la evaluación del nivel de eficacia y satisfacción del desarrollo del Programa de Ayuda.

La medición de la incidencia del bullying en los grupos se realizó a través del Insebull ${ }^{1}$, mediante un autoinforme y un heteroinforme. El autoinforme (Ortega y Avilés, 2005) fue validado mediante el método de componentes principales no lineales (princals) y por el juicio de expertos (Alpha=,9634). Su fiabilidad obtuvo un Alpha de Crombach de .8678 de consistencia interna. Las dimensiones de los items que mide el instrumento quedan recogidas en el Tabla $n^{\circ} 4$.

Para las valoraciones de la satisfacción de los diferentes miembros de la comunidad educativa sobre el Programa de Ayuda en el centro 1, se utilizó el método descriptivo basado en el autoinforme o cuestionario. Se pasaron diversos cuestionarios integrados por preguntas abiertas y cerradas de opción múltiple. En la Tabla 5 exponemos el contenido de las preguntas formuladas a cada uno de los participantes descritos.

\footnotetext{
${ }^{1}$ El Insebull (Avilés y Elices, 2007) es una aplicación informática que integra un autoinforme (Ortega y Avilés, 2005) de 36 items y un sociograma del maltrato (Avilés y Elices, 2005)
} 
Tabla 4. Dimensiones que miden los items del autoinforme

\begin{tabular}{|c|c|}
\hline VARIABLE & EXPLICACIÓN \\
\hline EDAD & Edad de los sujetos \\
\hline CENTRO & Centro Educativo \\
\hline NIVEL & Nivel Educativo \\
\hline CLASE & Grupo Clase \\
\hline AMBITO & Ámbito Rural o Urbano \\
\hline GÉNERO & Sexo \\
\hline $\mathrm{N}^{\circ} \mathrm{HNOS}$ & $\mathrm{N}^{\circ}$ Hermanos/as \\
\hline TIPINT & Tipos de Intimidación \\
\hline RELCOMPA & Relación con compañeros/as \\
\hline CANTIAMI & Cantidad de amigos/as \\
\hline SOLRECRE & Estar solo/a en recreo \\
\hline SENTIES & Cómo se siente el sujeto en el IES \\
\hline TRATOPRO & Trato percibido del Profesorado \\
\hline SENTICAS & Cómo se siente el sujeto en su casa \\
\hline MODELFAM & Modelo de composición familiar \\
\hline MIEDOIES & Frecuencia en sentir miedo en IES \\
\hline CAUSMIE1 & Causas del Miedo: No lo siente \\
\hline CAUSMIE2 & Causas del Miedo : El Profesorado \\
\hline CAUSMIE3 & Causas del Miedo: Los/as compañeros/as \\
\hline CAUSMIE 4 & Causas del Miedo: No saber hacer las cosas \\
\hline CAUSMIE5 & Causas del Miedo: otras \\
\hline INRECICU & Frecuencia de intimidaciones recibidas por curso \\
\hline DURAINTI & Duración de la intimidación \\
\hline SENTIVIC & Sentimientos de la víctima \\
\hline CAUSVIC1 & Atribución causal de la víctima: Nadie me intimida \\
\hline CAUSVIC2 & Atribución causal de la víctima: No lo sabe \\
\hline CAUSVIC3 & Atribución causal de la víctima: Por provocar yo \\
\hline CAUSVIC4 & Atribución causal de la víctima: Por ser diferente \\
\hline CAUSVIC5 & Atribución causal de la víctima: Por ser más débil \\
\hline CAUSVIC6 & Atribución causal de la víctima: Por molestarme \\
\hline CAUSVIC7 & Atribución causal de la víctima: Por gastarme una broma \\
\hline CAUSVIC8 & Atribución causal de la víctima: Por merecérmelo \\
\hline CAUSVIC9 & Atribución causal de la víctima: Otras \\
\hline UBIAGRE1 & Ubicación de los agresores/as: En el mismo grupo \\
\hline UBIAGRE2 & Ubicación de los agresores/as: En el mismo nivel \\
\hline UBIAGRE3 & Ubicación de los agresores/as: En nivel superior \\
\hline UBIAGRE4 & Ubicación de los agresores/as: En nivel inferior \\
\hline UBIAGRE5 & Ubicación de los agresores/as: No sabe \\
\hline IDENTAGR & Identificación de los agresores/as en sexo y número \\
\hline LOCIN1 & Localización del maltrato: En clase con profesorado \\
\hline LOCIN2 & Localización del maltrato: En clase sin profesorado \\
\hline LOCIN3 & Localización del maltrato: En los pasillos \\
\hline LOCIN4 & Localización del maltrato: En los aseos \\
\hline LOCIN5 & Localización del maltrato: En el patio con profesorado \\
\hline LOCIN6 & Localización del maltrato: En el patio sin profesorado \\
\hline LOCIN7 & Localización del maltrato: En alrededores del IES \\
\hline LOCIN8 & Localización del maltrato: En la calle \\
\hline QUIENINT & Identificación de quien interviene para parar el maltrato \\
\hline DICEVIC1 & A quién se lo cuenta la víctima: Nadie me maltrata \\
\hline DICEVIC2 & A quién se lo cuenta la víctima: A nadie \\
\hline DICEVIC3 & A quién se lo cuenta la víctima: Al profesorado \\
\hline DICEVIC4 & A quién se lo cuenta la víctima: A mi familia \\
\hline DICEVIC5 & A quién se lo cuenta la víctima: A compañeros/as \\
\hline AGRPOTEN & Posibilidad de actuar como agresores/as potenciales \\
\hline
\end{tabular}




\begin{tabular}{|l|c|}
\hline SERAGRE0 & Frecuencia en ser maltratador \\
\hline SERAGRE3 & Frecuencia en ser agresor/a el último trimestre \\
\hline SENTIAGR & Sentimientos del agresor/a al maltratar \\
\hline CAUSAGR1 & Atribución causal del agresor/a: No maltrato a nadie \\
\hline CAUSAGR2 & Atribución causal del agresor/a: Por provocación \\
\hline CAUSAGR3 & Atribución causal del agresor/a: Porque otros me lo hacen a mí \\
\hline CAUSAGR4 & Atribución causal del agresor/a: Porque son diferentes \\
\hline CAUSAGR5 & Atribución causal del agresor/a: Porque son más débiles \\
\hline CAUSAGR6 & Atribución causal del agresor/a: Por molestar \\
\hline CAUSAGR7 & Atribución causal del agresor/a: Por bromear \\
\hline CAUSAGR8 & Atribución causal del agresor/a: Otros \\
\hline CRITAGR1 & Críticas percibidas por el agresor/a: No intimida a nadie \\
\hline CRITAGR2 & Críticas percibidas por el agresor/a: Nadie le dice nada \\
\hline CRITAGR3 & Críticas percibidas por el agresor/a: Del profesorado \\
\hline CRITAGR4 & Críticas percibidas por el agresor/a: De su familia \\
\hline CRITAGR5 & Críticas percibidas por el agresor/a: De sus compañeros/as \\
\hline CRITAGR6 & Refuerzo percibido por el agresor/a: Del profesorado \\
\hline CRITAGR7 & Refuerzo percibido por el agresor/a: De su familia \\
\hline CRITAGR8 & Refuerzo percibido por el agresor/a: De sus compañeros/as \\
\hline ESPETAGR & Percepción del agresor/a sobre la actitud de los espectadores/as \\
\hline ESPEFREC & Frecuencia de las intimidaciones desde los espectadores/as \\
\hline OPIAGRE & Opinión que tienen los espectadores/as sobre los agresores/as \\
\hline CAUSESP1 & Atribución causal de los espectadores/as: por molestar \\
\hline CAUSESP2 & Atribución causal de los espectadores/as: se meten con ellos \\
\hline CAUSESP3 & Atribución causal de los espectadores/as: son más fuertes \\
\hline CAUSESP4 & Atribución causal de los espectadores/as: por bromear \\
\hline CAUSESP5 & Atribución causal de los espectadores/as: otras \\
\hline ACTUESPE & Actuaciones de los espectadores/as ante las agresiones \\
\hline NECESOLU & Necesidad de solucionar el problema \\
\hline PROPSOLU & Propuestas para solucionar el problema \\
\hline REACCIONA & Reacción ante la intimidación \\
\hline DECANTA & Decantación moral ante la intimidación \\
\hline AUTOROL & Evaluación del rol personal \\
\hline
\end{tabular}

Tabla 5. Cuestiones a evaluar en el Programa de Ayuda del Centro y destinatarios del mismo

\begin{tabular}{|c|c|c|c|c|c|}
\hline & $\begin{array}{l}\text { Alumnado de } \\
\text { los equipos } \\
\text { de ayuda }\end{array}$ & $\begin{array}{l}\text { Alumnado } \\
\text { receptor del } \\
\text { servicio }\end{array}$ & $\begin{array}{c}\text { Padres de } \\
\text { alumnado de } \\
\text { equipos de } \\
\text { ayuda }\end{array}$ & $\begin{array}{l}\text { Docentes de } \\
\text { estos niveles }\end{array}$ & $\begin{array}{l}\text { Docentes } \\
\text { responsables } \\
\text { del Programa }\end{array}$ \\
\hline Conocimiento del Programa & - & - & $\cdot$ & $\cdot$ & \\
\hline Adecuación de la formación & - & & & & - \\
\hline $\begin{array}{l}\text { Frecuencia y tipología de los } \\
\text { casos tratados }\end{array}$ & - & & & & \\
\hline $\begin{array}{l}\text { Consecuencias, efectos (en el } \\
\text { mismo alumnado, en el gru- } \\
\text { po,...) }\end{array}$ & - & - & - & - & - \\
\hline $\begin{array}{l}\text { Valoración de ser integrante } \\
\text { de un equipo de ayuda }\end{array}$ & - & - & & & \\
\hline $\begin{array}{l}\text { Valoración de la eficacia de } \\
\text { la intervención }\end{array}$ & - & - & & & . \\
\hline $\begin{array}{ll}\text { Perspectivas, } & \text { continuidad } \\
\text { del Programa } & \\
\end{array}$ & $\cdot$ & $\cdot$ & $\cdot$ & " & - \\
\hline
\end{tabular}




\section{Análisis Estadístico}

El procesamiento de los datos se realizó utilizando el paquete estadístico SPSS 15.0. Se realizaron diversos análisis. Para la incidencia del maltrato en los diversos grupos se hicieron análisis de contingencia entre variables relevantes en los grupos analizados.

Para conocer el nivel de satisfacción de los diversos miembros de la comunidad educativa, se vaciaron los datos de los cuestionarios y se realizó un análisis descriptivo de los resultados. Al valorar las respuestas de estos cuestionarios, es necesario tener en cuenta que la intervención de los Equipos de Ayuda se realiza bajo el principio de confidencialidad por lo que, en ocasiones, algunas de sus actuaciones sólo son conocidas por los profesores responsables de realizar el seguimiento, pasando quizá totalmente desapercibidas para el resto de alumnado o profesorado.

\section{Procedimiento}

Se procedió a pasar el autoinforme sobre maltrato, a los tres grupos del centro 1 y a tres grupos del centro 2 en enero y junio del año 2007. Los grupos considerados en el centro 1 , junto con el resto de sus compañeros de segundo y tercero, participaron, durante el tiempo transcurrido entre las dos mediciones, en la puesta en marcha y desarrollo de un Programa de Ayuda. Los Equipos de Ayuda y el profesorado responsable recibieron la formación necesaria para el desarrollo del programa. En el centro 2 no se aplicó.

Los cuestionarios para la evaluación del programa fueron administrados de la siguiente manera: al alumnado en una sesión de tutoría, a los padres se les hicieron llegar a través de sus hijos/as, y a los profesores en su casillero personal. Todos ellos fueron cumplimentados de forma anónima. En el caso del alumnado, la única identificación fue el nivel y grupo al que correspondían, ya que nos interesaba conocer si existían o no diferencias en las valoraciones en función de la edad o nivel educativo del alumno o en relación con otras variables que pudieran caracterizar a cada grupo. Por lo tanto, podemos decir que la participación del alumnado fue "obligada", mientras que para los docentes de los grupos implicados $\left(1^{\circ}, 2^{\circ}\right.$ y $3^{\circ}$ de la ESO) fue voluntaria. Consideramos que el porcentaje de participación es un indicador del interés por el Programa. 


\section{Resultados}

\section{Respecto al bullying y su incidencia}

En primer lugar, se realizó un contraste de medias para grupos independientes, con el fin de establecer las diferencias a través de la comparación de las varianzas de los grupos de los dos centros. Los resultados de la prueba de Levene, para ambos centros, en los factores significativos del maltrato, no señalaron diferencias significativas en ninguno de los factores analizados. Esto nos indica que en principio los grupos son iguales y por tanto se pueden hacer comparaciones fiables entre ellos. La información se refleja en la Tabla 6 .

Tabla 6. Igualdad de las varianzas ${ }^{4}$

\begin{tabular}{|c|c|c|c|c|c|c|}
\hline \multicolumn{7}{|c|}{$\begin{array}{l}\text { Prueba de muestras independientes } \\
\text { Prueba de Levene para la igualdad de varianzas }\end{array}$} \\
\hline & $\mathbf{F}$ & Sig. & $\mathbf{t}$ & gl & Sig. (bilateral) & $\begin{array}{l}\text { Diferencia } \\
\text { de medias }\end{array}$ \\
\hline Intimidación &, 002 & ,967 &,- 026 & 262 & ,980 &,- 033 \\
\hline $\begin{array}{l}\text { Carencia de } \\
\text { Soluciones }\end{array}$ & ,002 & 968, & ,073 & 262 & 942 & ,115 \\
\hline Victimización &, 001 & ,981 &,- 088 & 262 & ,930 &,- 135 \\
\hline $\begin{array}{l}\text { Inadaptación } \\
\text { Social }\end{array}$ &, 028 & ,866 &,- 316 & 262 & ,753, &,- 352 \\
\hline $\begin{array}{l}\text { Constatación } \\
\text { del Maltrato }\end{array}$ & ,006 & ,938 & ,104 & 262 & ,917 & ,084 \\
\hline $\begin{array}{l}\text { Identificación } \\
\text { del Maltrato }\end{array}$ &, 048 &, 826 &, 301 & 262 & ,763 & ,295 \\
\hline $\begin{array}{l}\text { Vulnerabili- } \\
\text { dad Escolar }\end{array}$ &, 042 &, 839 &,- 002 & 262 & ,998 &,- 004 \\
\hline $\begin{array}{l}\text { Falta de Inte- } \\
\text { gración Social }\end{array}$ & ,044 &, 834 &, 092 & 262 & ,927 & ,075 \\
\hline $\begin{array}{l}\text { Total de la } \\
\text { Escala de } \\
\text { Previsión de } \\
\text { Maltrato }\end{array}$ &, 101 &, 751 &,- 056 & 262 & ,956 &,- 104 \\
\hline
\end{tabular}

Se han realizado también pruebas entre los grupos de alumnos/as que realizaron el Programa de Ayuda (los del Centro 1) para determinar su igualdad, con el fin de matizar o precisar las conclusiones acerca de los efectos del Programa. Se compararon sus medias, no encontrándose diferencias significativas, conforme aparece en la Tabla $n^{0} 7$. Los grupos com- 
parados se muestran iguales y esto significa que las posibles diferencias que puedan aparecer entre ellos en la medida post, se deban al tratamiento aplicado.

Tabla 7. Tabla de Anova.

\begin{tabular}{|l|c|c|c|c|}
\hline & \multicolumn{2}{|c|}{$\begin{array}{c}\text { Datos inter- } \\
\text { grupos Centro } \\
1 . \text { Medida pre }\end{array}$} & $\begin{array}{c}\text { Datos inter- } \\
\text { grupos Centros } \\
1 \text { y 2 medida } \\
\text { post }\end{array}$ \\
\hline & F & Sig. & F & Sig. \\
\hline INTIMIDACIÓN * GRUPO &, 097 &, 908 &, 011 &, 915 \\
\hline CARENCIA DE SOLUCIONES * GRUPO &, 512 &, 602 &, 009 &, 923 \\
\hline VICTIMIZACIÓN * GRUPO &, 296 &, 745 &, 020 &, 887 \\
\hline INADAPTACIÓN SOCIAL * GRUPO &, 202 &, 818 &, 078 &, 780 \\
\hline CONSTATACIÓN DEL MALTRATO * GRUPO &, 482 &, 620 &, 002 &, 964 \\
\hline IDENTIFICACIÓN DEL MALTRATO * GRUPO &, 937 &, 397 &, 096 &, 757 \\
\hline VULNERABILIDAD ESCOLAR * GRUPO &, 309 &, 735 &, 025 &, 875 \\
\hline FALTA DE INTEGRACION SOCIAL * GRUPO &, 405 &, 669 &, 000 &, 987 \\
\hline $\begin{array}{l}\text { TOTAL ESCALA DE } \\
\text { PREVISIÓN DE MALTRATO * GRUPO }\end{array}$ &, 016 &, 984 &, 004 &, 950 \\
\hline
\end{tabular}

Finalmente, se compararon las muestras de los dos centros con el fin de valorar las diferencias entre las puntuaciones obtenidas en los dos momentos de medición. Los análisis indican ausencia de diferencias significativas entre los resultados de los dos centros, conforme aparece en la Tabla 7, en los factores analizados.. Esto indica que la aplicación del Programa en el centro 1 no aporta diferencias significativas en los factores analizados respecto a los resultados del centro 2 .

Sin embargo, al analizar los items mediante la prueba $\mathrm{T}$ de diferencia de medias, encontramos diferencias significativas entre la medida pre y la medida post en los ítems referidos a percepción de frecuencia de bullying desde la posición de agresor/a, como se indica en la Tabla 8.

Por otra parte, en el análisis de contingencias observamos que es la percepción de frecuencia reconocida por los agresores, el ítem que puede marcar alguna tendencia hacia diferencias significativas $(p \leq .05)$ respecto a los dos momentos de medida. Interpretaríamos que tras la aplicación del Programa los agresores reconocen que agreden más, quizás por efecto de la puesta en marcha del mismo, lo que les hace tomar más conciencia del problema (ver Tabla 9). Además, observamos una correlación negativa significativa $(\mathrm{r}=-.204 ; p \leq .05)$ entre las va- 
riables "momento de medida" y "comunicación de los hechos a los compañeros/as por parte de la víctima". Esta correlación se muestra como tendencia en lo que hace referencia al maltrato social percibido por los sujetos, después de finalizada la intervención $(\mathrm{r}=-.154 ; p \leq .05)$. Es decir, conforme se ha puesto en marcha el Programa de Ayuda, son más las víctimas que cuentan a los compañeros sus problemas relacionados con el bullying y se hace más consciente el maltrato de tipo social.

Tabla 8. Diferencias de medias

\begin{tabular}{|l|c|c|}
\hline \multicolumn{1}{|c|}{ Items } & Dif. Medias & Sig. \\
\hline CENTRO EDUCATIVO &, 035 &, 573 \\
\hline INSULTAR, PONER MOTES &, 287 &, 133 \\
\hline REIRSE DE ALGUIEN, DEJAR EN RIDÍCULO &, 074 &, 642 \\
\hline HACER DAÑO FÍSICO &, 331 &, 097 \\
\hline HABLAR MAL DE ALGUIEN &, 181 &, 298 \\
\hline AMENAZAR, CHANTAJEAR &, 238 &, 170 \\
\hline RECHAZAR, AISLAR &, 372 &, 062 \\
\hline OTRAS FORMAS &, 481 &, 298 \\
\hline INCIDENCIA DEL BULLYING DESDE LAS VÍCTIMAS &, 121 &, 080 \\
\hline FRECUENCIA DEL BULLYING DESDE QUIENES AGREDEN &, 141 &, 050 \\
\hline FRECUENCIA DEL BULLYING DESDE LOS ESPECTADORES &, 387 &, 069 \\
\hline
\end{tabular}

Tabla 9. Análisis de contingencia del item 22 del autoinforme (Ortega y Avilés, 2005)

\begin{tabular}{|c|c|c|c|c|c|c|c|c|c|}
\hline \multicolumn{10}{|c|}{$\begin{array}{l}\text { Frecuencia percibida del bullying desde quienes agreden } \\
\text { (al trimestre) }\end{array}$} \\
\hline & & & Perdidos & Nunca & $\begin{array}{l}\text { Menos de } \\
5 \text { veces }\end{array}$ & $\begin{array}{l}\text { Entre } 5 \text { y } \\
10 \text { veces }\end{array}$ & $\begin{array}{l}\text { Entre } 10 \\
\text { y } 20 \text { veces }\end{array}$ & $\begin{array}{l}\text { Más de } 20 \\
\text { veces }\end{array}$ & Total \\
\hline \multirow[t]{2}{*}{ MOMENTO } & 1 & Recuento & 0 & 120 & 10 & 0 & 1 & 1 & 132 \\
\hline & & $\%$ de ITEM 22 &, $0 \%$ & $53,3 \%$ & $33,3 \%$ &, $0 \%$ & $50,0 \%$ & $33,3 \%$ & $50,0 \%$ \\
\hline \multirow[t]{2}{*}{ MOMENTO } & 2 & Recuento & 1 & 105 & 20 & 3 & 1 & 2 & 132 \\
\hline & & $\%$ de ITEM 22 & $100,0 \%$ & $46,7 \%$ & $66,7 \%$ & $100,0 \%$ & $50,0 \%$ & $66,7 \%$ & $50,0 \%$ \\
\hline \multirow{2}{*}{\multicolumn{2}{|c|}{ Total }} & Recuento & 1 & 225 & 30 & 3 & 2 & 3 & 264 \\
\hline & & $\%$ de ITEM 22 & $100,0 \%$ & $100,0 \%$ & $100,0 \%$ & $100,0 \%$ & 100,0 & $100,0 \%$ & 100,0 \\
\hline
\end{tabular}


Respecto a la satisfacción de los usuarios y destinatarios del Programa de Ayuda en el seno de la comunidad educativa

Los resultados más relevantes son los siguientes:

1) Todos los sectores de la comunidad educativa consideran., en mayor o menor medida, que el Programa es válido para lograr los objetivos que persigue (ver Figura 1).

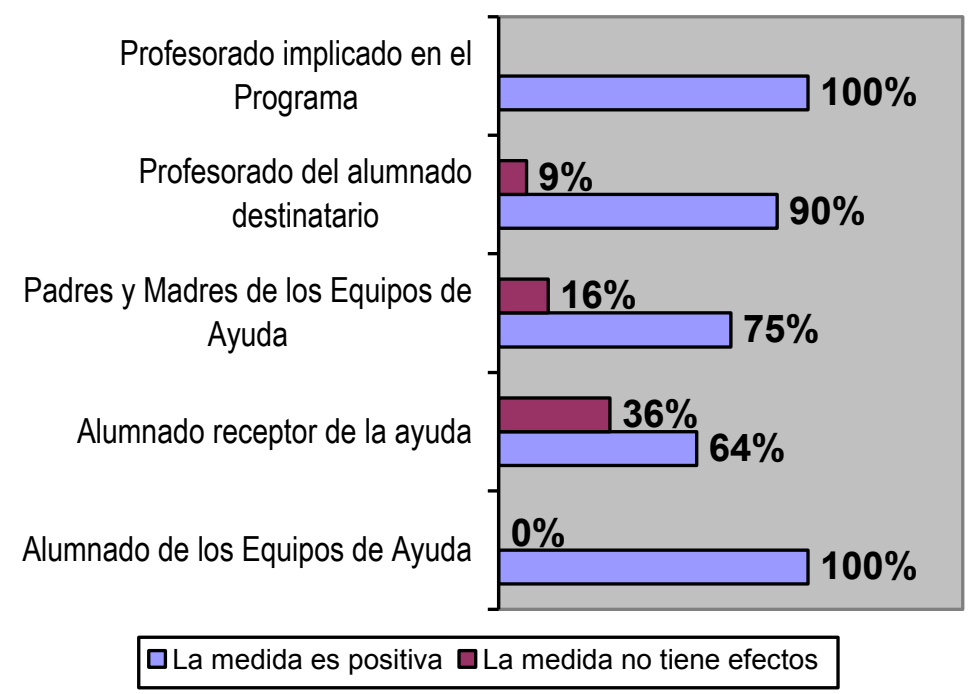

Figura 1. Valoración del Programa de Ayuda en los sectores de la comunidad educativa

2) El alumnado integrante de los Equipos de Ayuda y sus padres atribuyen al programa el desarrollo de algunas habilidades de ayuda, como son la empatía, la escucha activa y la capacidad para reflexionar antes de actuar, conforme se refleja en la Figura 2.

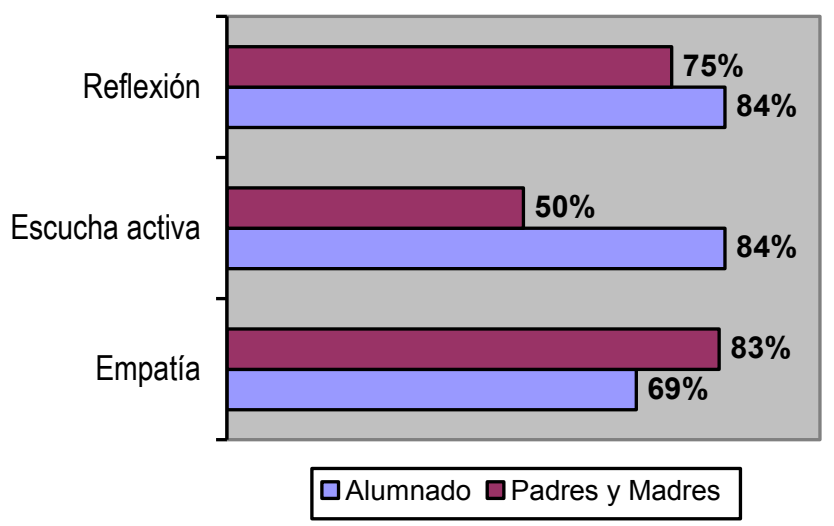

Figura 2. Valoración de algunas habilidades desarrolladas en el Programa de Ayuda 
3) Como puede observarse en la Figura 3, todos los sectores creen que el programa debería continuar en el centro.

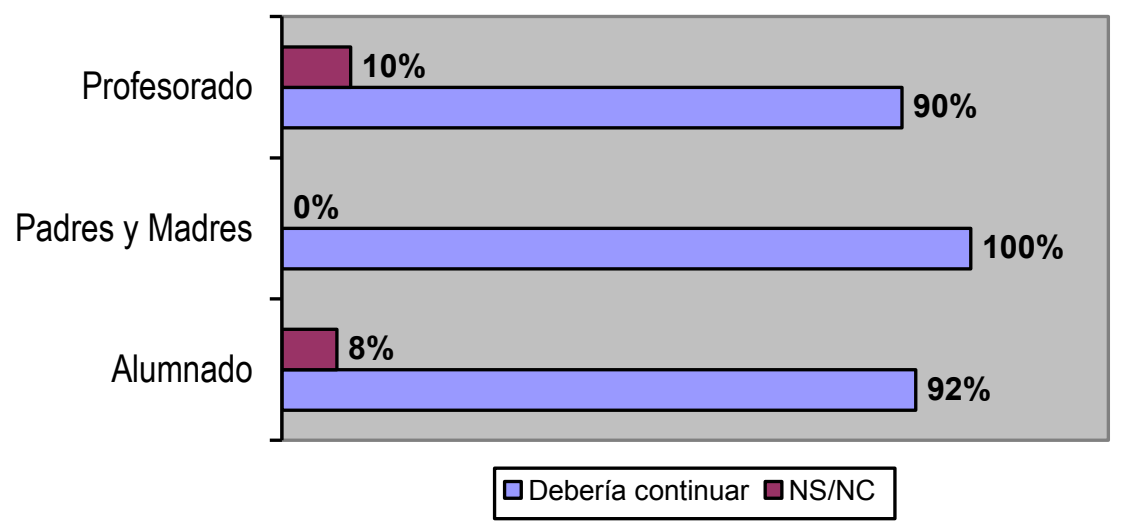

Figura 3. Valoración de la continuidad del Programa de Ayuda

4) Destacamos a continuación otras valoraciones relevantes de cada sector de la comunidad educativa:

Del alumnado integrante de los Equipos de Ayuda:

Un $84 \%$ cree que formar parte de un Equipo de Ayuda ha modificado su forma de pensar, de actuar y de analizar situaciones, aludiendo a cambios como los siguientes: "ahora me fijo más en el comportamiento de mis compañeros", "me siento más capacitado para ayudar", "escucho mejor a los demás", "me pongo más en el lugar del otro". Un 75\% cree que le ha ayudado a mejorar la comunicación con los otros.

El 69\% ha intervenido en alguna ocasión. Los casos en los que han trabajado han sido mayoritariamente sobre problemas personales de los compañeros, seguidos de acompañamiento a chicos/as que estaban solos y en tercer lugar en situaciones de conflicto entre compañeros.

Ningún miembro de Equipo de Ayuda dice haber sentido algún tipo de discriminación -en sentido negativo- por parte de sus compañeros.

Un $76 \%$ valora su intervención como medianamente eficaz y el resto, un $24 \%$, como muy eficaz.

Del alumnado que recibe el servicio de ayuda:

El 64\% considera positivo que exista un Equipo de Ayuda en su clase, ninguno lo considera negativo y el $36 \%$ contesta que no lo considera ni positivo ni negativo. En $1^{\mathrm{o}}$ de ESO es mayor el porcentaje que lo valora positivamente, y en $2^{\circ}$ de ESO se hace esta consideración 
en menor proporción. Seguramente estos datos revelan la influencia de las expectativas y opiniones de los tutores. Observamos que existe una relación directa entre la naturaleza de éstas y las que genera su alumnado.

Ha solicitado ayuda un 3\% de esta población. Un $65 \%$ no, pero lo haría si lo necesitara, y un $32 \%$ no lo haría nunca ("porque prefieren acudir a sus amigos”). Estos resultados son similares en cada uno de los tres niveles educativos.

De las familias del alumnado del Equipo de ayuda.

El 75\% considera que formar parte de un Equipo de Ayuda ha sido una experiencia positiva para su hijo, un $16 \%$ considera que no ha tenido efecto y el resto, no contesta. El $33 \%$ considera que ha ayudado a su hijo/a a mejorar su formación como persona, el $25 \%$ a ser más sensible ante las dificultades de los demás, y otro $25 \%$ a ser más reflexivo antes de actuar. Ningún padre o madre ha detectado aspectos negativos en el desarrollo de este programa.

Del profesorado de $1^{\circ}, 2^{\circ}$ y $3^{\circ}$ de la ESO:

El $50 \%$ del profesorado considera que ha tenido efectos beneficiosos para todo el alumnado. Un 22\% cree que sólo en quienes integran los Equipos de Ayuda y un 9\% opina que no ha tenido ningún efecto.

El $63 \%$ no ha detectado aspectos negativos en el desarrollo de este programa, un $22 \%$ no ha opinado y un 13\% sí ha identificado cuestiones negativas; entre ellas citan: falta de sustitución del profesorado que ha impartido la formación, la pérdida de clase de los alumnos durante ese periodo, el procedimiento seguido para la selección de los Equipos de Ayuda.

Del equipo de profesores implicados directamente en el programa:

El $100 \%$ realiza una valoración global muy positiva del programa, especialmente aquellos que por primera vez se han acercado a estos temas.

El 100\% considera que ellos mismos han ampliado su formación sobre cuestiones relacionadas con la convivencia. 


\section{Discusión y Conclusiones}

Los resultados de la investigación confirman lo que otras investigaciones han encontrado respecto a programas que ponen en juego estrategias de cooperación y ayuda en el aprendizaje (Cowie, Smith, Boulton y Laver, 1994) y respecto a su influencia en la reducción de la incidencia del bullying (Naylor y Cowie, 1999; Whitney y Smith, 1993) y de la actuación de los agresores/as.

Parece que los datos nos indican que la puesta en marcha del Programa de Ayuda no ha servido específicamente para reducir la incidencia del bullying. Los niveles de percepción de bullying son similares en el centro 1 después de haber sido aplicado el Programa de Ayuda, si atendemos a lo que responden víctimas y espectadores del maltrato.

Sin embargo, se observan diferencias significativas en la percepción que tienen los agresores/as de sus actuaciones antes y después de aplicar el Programa. Esta nueva aportación resulta relevante ya que nos habla de que el Programa contribuye a la toma de conciencia en los agresores/as de sus actuaciones (Menesini et al., 2003). Esto permitiría articular posibles actuaciones que puedan comprometerles en la resolución del bullying (Aviles, 2006; Pikas, 1989; Rigby, 1996). Aspecto éste, absolutamente crucial a la hora de decidir los modelos disciplinarios y normativos en el seno de la comunidad educativa. Además, este incremento de conciencia de los agresores/as frente a las situaciones de bullying puede ayudar a la efectividad de otros programas específicos encaminados a combatir el maltrato, aprovechando el clima de concienciación que genera el Programa de Ayuda.

Por otra parte, la puesta en marcha del Programa habría explicitado formalmente la preocupación de la comunidad educativa respecto al problema. Oficialmente se habla de ello, se trabaja para reducirlo y ayudar a quien pueda estar en riesgo o dificultades, etc. Existen figuras (Equipos de Ayuda) que son reconocidas, profesorado que coordina las tareas y realiza la formación, etc. Es decir, el lenguaje institucional de la comunicad educativa maneja el lenguaje de la "ayuda". Estructuralmente el Programa es reconocido y ocupa tiempos y espacios del centro (tutoría, reuniones de coordinación del profesorado, reuniones de Equipos de Ayuda, jornadas de formación, etc.) Este respaldo institucional contribuye, sin duda, a ese aumento de conciencia y/o preocupación por parte de todos los sectores de la comunidad educativa (Galloway y Roland, 2004). 
En relación con la idea anterior y/o como consecuencia de ella, se produce otro de los resultados relevantes de esta investigación, que coincide con otras (Cowie y Olafsson, 2000), y que tiene que ver con que hayan sido significativamente más las víctimas que se han decidido a contar los hechos después de desarrollarse el Programa. Esto sitúa al mismo como un instrumento útil para canalizar las necesidades de comunicación, entre otras, que tienen todas las víctimas. Sabemos de las dificultades que tienen las víctimas para comunicar lo que les pasa (Avilés, 2002; Ortega y Mora-Merchán, 2000; Shariff, 2008; Rigby, 2008). Otros programas de ayuda ya han puesto de manifiesto su efectividad para vencer algunos de los miedos que tienen las víctimas para confiarse a un igual (Fernández, Villoslada y Funes, 2002). Sin duda son los iguales dispuestos a ayudar, los amigos, quienes en mejor posición están para echar una mano cuando hace falta. Sin embargo, también son importantes medidas institucionales para canalizar la comunicación de las victimas: correos electrónicos, una página web que respetando el anonimato pueda recoger los miedos de quien está siendo acosado, incluso la teleayuda (Cowie y Fernández, 2006); y, sin duda, las redes estables de ayuda, constituidas y fomentadas por los adultos de la comunidad educativa, como son los Equipos de Ayuda, que con respaldo institucional y formalmente constituidos, pueden colaborar a que las víctimas visualicen la ayuda a la que pueden acudir cuando lo necesiten.

Por último, respecto al maltrato social (rechazar, aislar, no juntarse con alguien, no dejar participar), la aplicación del Programa de Ayuda ha servido para concienciar al alumnado sobre este tipo de maltrato y para rebajar significativamente su incidencia entre las medidas pre y post. Este resultado nos parece relevante, dado que el maltrato social no es, en el tramo de edad estudiado, precisamente de los de mayor incidencia y reconocimiento entre el alumnado (Avilés, 2002, Ortega y Mora-Merchán, 2000). Pensamos que la puesta en marcha del Programa ha obligado a reflexionar sobre todas las formas de maltrato posibles, incluso aquellas que pueden quedar más ocultas o pasar desapercibidas, y que no por eso son menos graves o dañinas. Valoramos esta contribución como muy positiva.

Sin embargo, no se producen variaciones respecto a la percepción de incidencia que el alumnado tiene del maltrato verbal o del maltrato físico, después de la aplicación del Programa, datos coincidentes con los de otras investigaciones (Cowie y Fernández, 2006; Cowie y Olafsson, 2000). 
Las conclusiones de la investigación apuntan a la necesidad de incorporar estrategias específicas en la comunidad educativa dirigidas a erradicar el maltrato al mismo tiempo que se ponen en marcha los Programas de Ayuda. Deben establecerse planes de colaboración entre familias, alumnado y profesorado para definir qué entiende por maltrato cada sector de la comunidad educativa; determinar procedimientos para medir exactamente el bullying y saber cuánto sucede y de qué calidad es; ofertar espacios y tiempos para compartir preconcepciones y formación sobre el tema, en escuelas de habilidades parentales, por ejemplo; integrar al alumnado y a las familias en la puesta en marcha de medidas antibullying que deberá impulsar el profesorado, en actividades de acompañamiento, supervisión, observación y convivencia en recreos, entradas y salidas, actividades extraescolares y paraescolares; etc. Organizativamente, constituir un grupo de trabajo antibullying donde estén todos los sectores de la comunidad educativa representados, organizar mejor las tutorías, establecer criterios para los agrupamientos del alumnado, elaborar mapas de riesgos en el centro, decidir los protocolos de actuación cuando se sabe que ha sucedido un caso de acoso, coordinar las decisiones de los equipos docentes en cada grupo-clase, unificar las actuaciones contra el bullying por parte de todos los adultos que conviven con el alumnado,... Desde el punto de vista curricular, hablar de esto en la clase, en todas las materias, en tutoría, utilizar metodologías participativas y favorecedoras para el tratamiento del problema, con el uso del role play, las dramatizaciones, el análisis de casos, los talleres de prensa,... Elaborar respuestas para cuando surjan los hechos y el debate en clase, saber qué decir la primera vez, saber cómo dirigirse a los agresores/as, saber en qué aspectos incidir y cuáles destacar, dejar clara ante la clase una actitud inequívoca ante el maltrato y todas sus formas, ... En el grupo de convivencia habitual, establecer una dinámica relacional clara con el alumnado, en la forma de resolver los conflictos entre alumnado y profesorado; saber y conocer las redes afectiva, de poder y de tareas vigente en el grupo-clase; trabajar con el grupo la cultura del chivato, fomentando los valores de ayuda frente a los del abuso; establecer y propiciar que el alumnado fije las normas antibullying en el seno del grupo-clase; construir estructuras y redes de ayuda como los Equipos de Ayuda con el objetivo de visualizar los valores de la cooperación, la colaboración, la ayuda o el consuelo. Establecer programas de autoayuda, asertividad y autoafirmación para las víctimas, fomentar programas de control de la ira y la agresividad para agresores/as y programas de resolución colaborativa de los conflictos y de desarrollo moral entre los espectadores/as.

Sin duda, todas ellas han de ser iniciativas combinadas, duraderas, planificadas y colectivas (Smith, Pepler y Rigby, 2004) que discriminen el abordaje del bullying en el marco 
de un Programa Antibullying respaldado y consensuado por la comunidad educativa. Medidas que resultarán poderosas y consistentes (Sullivan, Cleary y Sullivan, 2004) para reducir la incidencia del maltrato entre iguales en los contextos grupales de convivencia entre pares. 


\section{Referencias}

Avilés, J. M. (2002). La intimidación entre iguales (bullying) en la Educación Secundaria Obligatoria. Validación del Cuestionario CIMEI y estudio de incidencia. Tesis doctoral no publicada. Universidad de Valladolid.

Avilés, J.M. (2006a). Bullying: el maltrato entre iguales. Agresores, víctimas y testigos en la escuela. Salamanca: Amarú.

Avilés, J. M. (2006b) Diferencias de atribución causal en el bullying entre sus protagonistas. Electronic Journal of Research in Educational Psychology, 4 (2) 136-148

Avilés, J. M. (2006c) L'assetjament escolar. Orientacions per a la prevenció i la intervenció. Guia del professorat. Palma de Mallorca: Consejería de Trabajo y Stei-i.

Avilés, J. M. y Alonso, M. N. (2008). Análisis de componentes identificativos de la violencia en el contexto excolar.Violencia, conflicto y maltrato.Itinerario de frontera. En I. Leal, J.L. Pais, I. Silva y S. Marques, (Eds.), $7^{\circ}$ Congresso Nacional de psicología da saúde, (pp. 119-129). Porto: ISPA Ediciones.

Avilés, J. M. y Elices, J. A. (2007). Insebull, instrumentos para la evaluación del bullying. Madrid: CEPE.

Avilés, J. M. y Mascarenhas, S. (2007). Bullying - Agresividad, conflicto y violencia interpersonal. Diferencias de atribución causal de sus protagonistas en enseñanza secundaria obligatoria de España (Valladolid) y Brasil (Amazonas/Humaitá). En A. Barca, M. Peralbo, A. Porto, B. Duarte da Silva y L. Almeida (Eds.), IX Congreso internacional galego-portugués de psicopedagogía, (pp. 141-153). A Coruña: Universidad de A Coruña y Universidad del Miño (Braga/Portugal).

Cowie, H. (1998). Perspectiva of teachers and pupils on the experience of peer support against bullying. Educational Research and Evaluation. U, 108-125.

Cowie, H. y Fernández, F.J. (2006). Ayuda entre iguales en las escuelas: desarrollo y retos. Electronic Journal of Research in Educational Psychology, 4 (2), 291-310

Cowie, H. y Jennifer, D. (2006) (Coords.). Ethical issues and children's rights. (UNIT C1). www.vista-europe.org/unit C1. (15 enero 2007).

Cowie, H. y Jennifer, D. (2007). Managing violence in schools. A whole-school approach to best practice. London: Paul Chapman Educational Publishing.

Cowie, H. y Olafsson, R. (2000). The role of peer support in helping the victims of bullying in a school with high levels of aggression. En School Psychology International, 21, 79-95.

Cowie, H., Smith, P., Boulton, M. y Laver, R. (1994). Cooperation in the multi-ethnic classroom. London: David Fulton. 
Fernández, I. Villoslada, E. y Funes, S. (2002). Conflicto en el centro escolar. Madrid: Catarata.

Galloway, D. y Roland, E. (2004). Is the direct approach to reducing bullying always the best? En P.K. Smith, D. Pepler y D. Rigby (Eds.) Bullying in schools. How successful can interventions be? (pp.37-55), Cambridge: Cambridge University Press.

Menesini, E., Codecasa, E., Benelli, B. y Cowie, H. (2003). Enhancing children's responsibilitiy to take action against bullying: evaluation of a befriending intervention in Italian middle schools. En Aggressive Behavior, 29, 1-14.

Naylor, P. y Cowie, H. (1999). The effectiveness of peer support systems in challenging school bullying: the perspectives and experiences of teachers and pupils. Journal of Adolescence, 22, 467-479.

Newman, R.S., y Murray, B.J. (2005). How students and teachers view the seriousness of peer harassment: when is it appropriate to seek help? En Journal of edutional psychology, 97 (3), 347-365

Ortega, R. y Avilés, J. M. (2005). Autoinforme para la evaluación del bullying. Valladolid: Autores.

Ortega, R. y Mora-Merchán, J. A. (2000). Violencia escolar: Mito o Realidad. Sevilla: Mergablum, Edición y Comunicación.

Pikas, A. (1989). The common concern method for the treatment of mobbing. En Roland, E. y Munthe, E. (Eds.), Bullying: An international perspective. (pp. 91-105). London: David Fulton.

Rigby, K. (1996). Bullying in schools. And what to do about it. Londres: Jessica Kingsley.

Rigby, K. (2008). Children and bullying.. Victoria: Blackwell Publishing.

Salmivalli, C., Lagerspetz, K.M.J., Björkqvist, K., Österman, K. y Kaukiainen, A. (1996). Bullying as a group process. Participant roles and their relations to social status within the group. Aggressive Behavior, 22, 1-15.

Shariff, S. (2008). Cyber-bullying. New York: Routledge.

Sharpe, S. y Smith, P. K. (1994). Tackling bullying in your school. Routledge. London.

Sharp, S., Cowie, H. y Smith, P. K. (1994). How to respond to bullying. En S.Sharp y P.K. Smith (Eds) Tackling bulling in your school, London: Routledge.

Smith, P. K. y Sharpe, S. (1994). School bullying. Insights and perspectives. Routledge. London. 
Smith, P. K., Pepler, D., Rigby, K. (2004). Bullying in schools. How successful can interventions $b e$ ? Cambridge: Cambridge University Press.

Sulllivan, K. (2001). The anti-bullying handbook. Auckland: Oxford University Press.

Sullivan, K., Cleary, M. y Sullivan, G. (2004). Bullying in secondary schools. London: Paul Chapman Publishing.

Trianes, M. V. (2005). Métodos didácticos para previr o acoso. En III xornadas Convivencia escolar "O acoso nas aulas". 20 y 21 de octubre de 2005. Pontevedra: Cruz Vermella Xuventude y Diputación Provincial.

Whitney, I. y Smith, P.K. (1993). A survey of the nature and extent of bullying in junior/-middle and secondary. En Educational Research, 35, 3-25. 